\title{
Demonstration of an erbium-doped microdisk laser on a silicon chip
}

\author{
T. J. Kippenberg* \\ Department of Applied Physics, California Institute of Technology, Pasadena, California 91125 USA \\ J. Kalkman and A. Polman ${ }^{\dagger}$ \\ Center for Nanophotonics, FOM-Institute AMOLF, Amsterdam, The Netherlands \\ K. J. Vahala \\ Department of Applied Physics, California Institute of Technology, Pasadena, California 91125 USA
}

(Received 1 August 2006; published 16 November 2006)

\begin{abstract}
An erbium-doped microlaser is demonstrated utilizing $\mathrm{SiO}_{2}$ microdisk resonators on a silicon chip. Passive microdisk resonators exhibit whispering-gallery-type modes (WGM's) with intrinsic optical quality factors of up to $6 \times 10^{7}$ and were doped with trivalent erbium ions (peak concentration $\sim 3.8 \times 10^{20} \mathrm{~cm}^{-3}$ ) using $\mathrm{MeV}$ ion implantation. Coupling to the fundamental WGM of the microdisk resonator was achieved by using a tapered optical fiber. Upon pumping of the ${ }^{4} I_{15 / 2} \rightarrow{ }^{4} I_{13 / 2}$ erbium transition at $1450 \mathrm{~nm}$, a gradual transition from spontaneous to stimulated emission was observed in the 1550-nm band. Analysis of the pump-output power relation yielded a pump threshold of $43 \mu \mathrm{W}$ and allowed measuring the spontaneous emission coupling factor: $\beta \approx 1 \times 10^{-3}$.
\end{abstract}

\section{DOI: 10.1103/PhysRevA.74.051802}

The increasing demand for computing power and communication bandwidth has generated an increased interest in the field of silicon photonics which aims at creating photonic elements utilizing standard, complementary metal-oxide semiconductor (CMOS) processing technology and materials, such as silica and silicon. As silica and silicon intrinsically lack direct optical transitions, alternative methods such as erbium doping or creating $\mathrm{Si}-\mathrm{SiO}_{2}$ nanostructures $[1,2]$ have been used to achieve optical emission. Despite these advances, achieving lasing in CMOS-compatible structures has remained challenging and has only been observed recently via Raman nonlinearity $[3,4]$ and by Er doping of silicon-chip-based silica toroid microcavities [5].

In this context, erbium is a particularly promising optical dopant as it provides gain in the $1.55-\mu \mathrm{m}$ telecommunication range and can be incorporated into a $\mathrm{SiO}_{2}$ by ion implantation [6], which is an inherently CMOScompatible process. However, due to the small emission cross section of Er at $1.55 \mu \mathrm{m}\left(\sigma=4 \times 10^{-21} \mathrm{~cm}^{2}\right)$ in conjunction with the fact that $\mathrm{Er}$ concentrations are limited to $N_{\mathrm{Er}} \sim 1 \times 10^{22}$ ions $/ \mathrm{cm}^{3}$ due to clustering, the modal gain is limited to approximately $\sim 7 \mathrm{~dB} / \mathrm{cm}$. Lasing action under these Er gain conditions requires optical resonators with quality factors $Q>\frac{2 \pi}{\lambda} n \frac{1}{\Lambda \sigma_{\varepsilon} N_{\mathrm{Er}}} \approx 10^{5}$ (assuming mode overlap $\Lambda=0.3$ and refractive index $n=1.44)$. These quality factors are readily available in toroidal microcavities [7] or silica microspheres [8], which use a laser-assisted reflow process to create ultrahigh- $Q$ cavities. Indeed, using these microcavity geometries rare-earth-doped microcavity lasers were first demonstrated $[5,9,10]$. However, the use of a $\mathrm{CO}_{2}$ laser reflow makes control through ion implantation difficult,

\footnotetext{
*Present address: Max Planck Institut für Quantenoptik, Garching, Germany

${ }^{\dagger}$ Electronic address: polman@amolf.nl

*Electronic address: vahala@its.caltech.edu
}

PACS number(s): 42.55.Sa, 42.60.Da, 81.16.Mk

since restructuring of the silica takes place. Increased control of the Er distribution relative to the optical mode is essential to achieve low lasing threshold or high gain. In addition, increased control is important in more complex materials systems such as, e.g., Er-doped silica codoped with $\mathrm{Si}$ nanocrystals that act as sensitizers for $\operatorname{Er}[11]$.

A more amenable geometry to these studies are planar microdisks $[12,13]$, which can be fabricated with small transverse dimensions on a $\mathrm{Si}$ chip. Fabrication of these disks does not rely on a laser reflow process, and doping with rare-earth ions or $\mathrm{Si}$ ions by ion implantation can be readily performed. Earlier work has already demonstrated that $Q$ $>10^{6}$ (at $1550 \mathrm{~nm}$ ) can be achieved in silica microdisks [12], which indicates the possibility to observe Er lasing in $\mathrm{SiO}_{2}$ microdisks.

In this Rapid Communication we demonstrate lasing in an Er-doped microdisk on a silicon chip. These microlasers combine modal engineering of microdisk resonators with the nanoscale precise control of the Er ion distribution in the disk through ion implantation, yielding optimum overlap of the Er ions with the fundamental whispering gallery modes (WGM's). By optical pumping of the Er ions at $1.48 \mu \mathrm{m}$ via a tapered optical fiber [14], lasing at $1.55 \mu \mathrm{m}$ was observed to occur at a threshold power of less than $43 \mu \mathrm{W}$. These results demonstrate Er lasing from a microdisk resonator using CMOS-compatible fabrication. Fabrication of Er-doped microdisk lasers proceeded in several steps. The substrate used in the present experiments was a $\mathrm{Si}(100)$ wafer covered with $1-\mu \mathrm{m}$-thick thermally grown $\mathrm{SiO}_{2}$ film. This oxide thickness represents a compromise between optical cavity design and ion distribution. The Er ions were incorporated in the $\mathrm{SiO}_{2}$ by $2-\mathrm{MeV}$ ion implantation at room temperature. The corresponding implantation range of $560 \mathrm{~nm}$ was chosen to obtain a good modal overlap with the fundamental WGM's (cf. Fig. 1). A total Er fluence of $4.2 \times 10^{15}$ ions $/ \mathrm{cm}^{2}$ ions was implanted, yielding a Gaussian depth distribution with a standard deviation $\sigma \approx 72 \mathrm{~nm}$. The average $\mathrm{Er}$ density within the implanted 


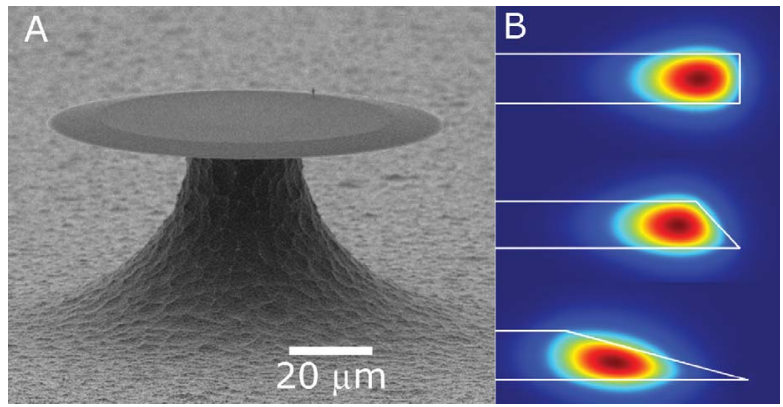

FIG. 1. (Color online) (a) Scanning electron micrograph image of a $60-\mu$ m-diameter silica microdisk cavity on a $\mathrm{Si}$ post on a silicon wafer. The wedge-shaped cavity boundary is intentionally induced during fabrication using a hydrofluoric acid etch. (b) A finite-element simulation of the intensity profile of the fundamental whispering gallery mode in a microdisk cavity (oxide thickness $1 \mu \mathrm{m}$, cavity radius $60 \mu \mathrm{m}, \lambda=1.54 \mu \mathrm{m}$ ) for three wedge angles: $90^{\circ}, 45^{\circ}$, and $22^{\circ}$. As can be evidenced in the simulation a progressive increase in the cavity boundary angle leads to radial shift of the mode towards the interior of the microdisk, thereby isolating the mode from the scattering-inducing cavity boundary.

layer is $N_{\mathrm{Er}}=3.8 \times 10^{20}$ ions $/ \mathrm{cm}^{3}$ (integrated over the full width at half maximum of the distribution), which corresponds to a modal gain of $2.66 \mathrm{~cm}^{-1}$ for $\sigma_{e}=7 \times 10^{-21} \mathrm{~cm}^{2}$. Upon implantation the oxidized wafer was annealed in Ar for $1 \mathrm{~h}$ at $800{ }^{\circ} \mathrm{C}$, which yielded optimized photoluminescence intensity and lifetime. The lifetime found ( $\sim 14 \mathrm{~ms})$ demonstrated successful passivation of implantation-induced defects. In addition, a reference sample was fabricated in which the Er implantation at an energy of $4 \mathrm{MeV}$ led to an implantation depth exceeding the oxide thickness of $1000 \mathrm{~nm}$. While this implantation depth precludes the observation of lasing, it served as a reference to assess any deterioration of the $Q$ factor due to ion implantation.

Following Er ion implantation, microcavity fabrication was carried out by first defining circular silica microdisks using optical lithography and hydrofluoric etching as detailed in Refs. [7,12]. The resulting microdisk had a diameter of $60 \mu \mathrm{m}$ as shown in the scanning electron micrograph (SEM) in Fig. 1. A key feature of the $\mathrm{SiO}_{2}$ disk, as seen in Fig. 1, is the strong inclination of the cavity sidewalls that is inherent to the fabrication process, which employs an isotropic HF etch. The disks were then undercut using a $\mathrm{XeF}_{2}$ gas to isotroptically etch the silicon and thereby create an air-clad whispering gallery mode structure. To optically test the microcavities, tapered optical fibers were used which provide high coupling ideality [14].

First, the reference microdisk resonators were tested in which no lasing of Er is expected. In these experiments the cavity modes typically appeared as doublets in the transmission spectrum, which is well known to result from scatteringinduced coupling of the clockwise and counterclockwise cavity modes $[15,16]$. An example of such a transmission spectrum is plotted as an inset in Fig. 2. Upon fitting the data the inferred $Q$ factor for each of the two modes was $5 \times 10^{7}$. This is a very high value for a planar microdisk, but still one order of magnitude lower than in the case of toroid microcavities [17]. Importantly, these $Q$ factors should
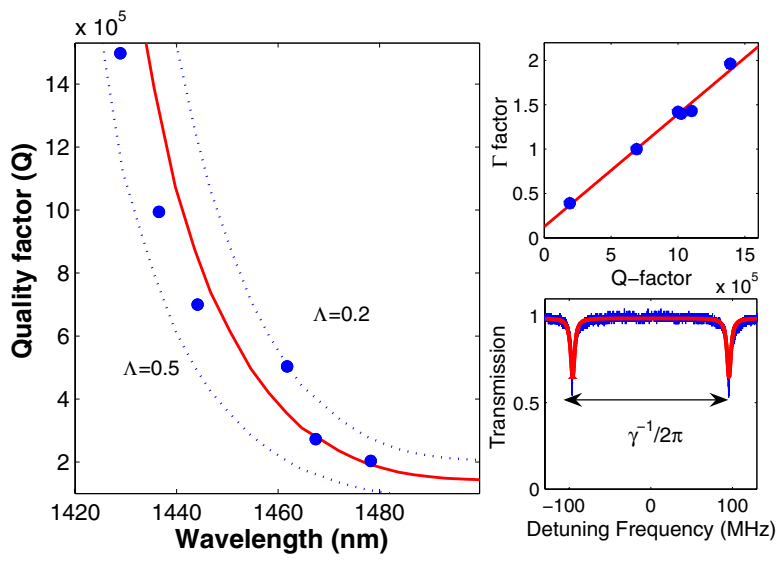

FIG. 2. (Color online) Experimentally measured quality factor $(Q)$ for subsequent WGM's in the weak pump regime. All modes belong to the same mode family (fundamental radial WGM's) and are separated by their different angular mode numbers. The solid line is a calculation taking into account wavelength-dependent absorption by Er, assuming $\Lambda=0.3$. Dotted lines show calculations for $\Lambda=0.2$ and $\Lambda=0.5$ for comparison. Lower right graph: a transmission spectrum exhibiting strong mode splitting (from a different sample). The upper right graph: the modal coupling parameteri.e., the ratio of the splitting frequency $\left(\gamma^{-1 / 2 \pi}\right)$ normalized with respect to the intrinsic cavity line width $\left(\tau^{-1} / 2 \pi\right)$-versus $Q$ for the modes measured in the main figure. The observed linear relationship demonstrates absorption-limited $Q$ behavior (solid line).

readily allow for observation of lasing of the Er ions. The observed high $Q$ is attributed to the wedged-shaped edge of the disk microcavity, which is believed to isolate modes from the disk perimeter and thereby reduce scattering losses [12]. This conjecture is further corroborated using numerical finite-element simulations as shown in Fig. 1, which demonstrate that an increased sidewall angle leads to an optical mode that is progressively more removed from the outer, lower cavity boundary (which can induce scattering losses). The $Q$ value of $5 \times 10^{7}$ also provides a lower bound on the effect of ion implantation induced defects.

Next, the 2-MeV Er-implanted microdisks, with an active Er distribution peaking at a depth of $560 \mathrm{~nm}$, were analyzed. First, the $Q$ factors in the range from 1410 to $1480 \mathrm{~nm}$ were measured for a microdisk with a diameter of $60 \mu \mathrm{m}$ (and an equivalent free spectral range, $F S R=9.1 \mathrm{~nm}$ ) as shown in Fig. 2. To avoid variations in the overlap factor and population-dependent $Q$, care was taken to measure the $Q$ at the same launched power for the same mode family (fundamental microdisk WGM). Upon approach of the $\mathrm{Er}^{3+}$ absorption band an increase in loss is evidenced. Specifically, while at $1428 \mathrm{~nm}$ the quality factor was observed to be $1.5 \times 10^{6}$ and it gradually reduced to a value as low as $2 \times 10^{5}$ at $1470 \mathrm{~nm}$. Both the absolute value of the $Q$ factor and its spectral dependence as shown in Fig. 2 are in good agreement with the theoretically predicted $\mathrm{Er}^{3+}$ absorption-limited $Q$ (solid red curve in Fig. 2) assuming $\Lambda=0.3$, a typical value for the fundamental WGM modes of the microdisk (cf. Fig. 1). Calculations for $\Lambda=0.2$ and $\Lambda=0.5$ are shown for comparison (dotted lines). The inset of Fig. 2 shows the $Q$ dependence of the modal coupling parameter [15] $(\Gamma=\tau / \gamma)$ defined as the ratio of the splitting frequency of the 

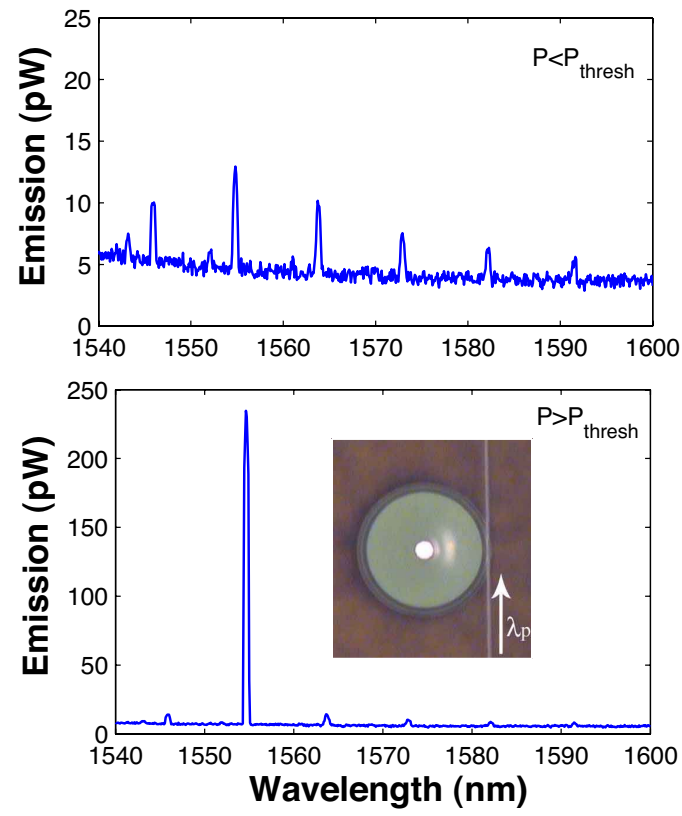

FIG. 3. (Color online) Upper graph: the subthreshold emission collected from the microdisk through the coupling fiber, when the cavity is resonantly pumped at $1480 \mathrm{~nm}$. Several fundamental cavity modes are observed (spaced with $\mathrm{FSR}=9.1 \mathrm{~nm}$ ). The weak, subsidiary peaks are attributed to fundamental modes of opposite polarization. The luminescence maximum at $1555 \mathrm{~nm}$ coincides with the peak of the erbium emission spectrum. Lower graph: the above-threshold spectrum in the presence of the 1450-nm pump. The nonlasing modes are suppressed by more than $15 \mathrm{~dB}$ in comparison with the lasing mode.

degeneracy-lifted cavity modes $\left(\gamma^{-1} / 2 \pi\right)$ and the intrinsic cavity linewidth $\left(\tau^{-1} / 2 \pi\right)$, both derived from the transmission spectra as in the inset of Fig. 2. Data are taken for several fundamental WGM's. The linear dependence that is observed demonstrates that the quality factors are dominated by absorption losses, in this case by $\mathrm{Er}^{3+}$ (i.e., since the scattering rate is observed to be nearly constant for all pump modes the variation of $\Gamma$ must be caused by a change in cavity absorption).

The excited erbium ions within the microcavity readily couple to the cavity modes. Due to the Stark broadening of ${ }^{4} I_{15 / 2} \rightarrow{ }^{4} I_{13 / 2}$ transitions of $\mathrm{Er}^{3+}$ in $\mathrm{SiO}_{2}$, the ions are coupled to many modes of the cavity. Indeed, as shown in Fig. 3, upon pumping at $1450 \mathrm{~nm}$ the spectrum collected through the coupled fiber taper contains several peaks throughout the erbium emission band (separated by the FSR of $9.1 \mathrm{~nm}$ ), clearly demonstrating that the erbium ions are coupled to the (fundamental) cavity modes. Weaker, subsidiary peaks observed in Fig. 3 are attributed to Er ions coupling to fundamental WGM's of opposite polarization. Note that the relative strength of the observed $\mathrm{Er}^{3+}$ peak emission collected in the tapered fiber depends both on the $\mathrm{Er}^{3+}$ emission spectrum and the wavelength-dependent ratio of internal to external quality factor, and therefore does not correspond exactly to the $\mathrm{Er}^{3+}$ emission cross-section spectrum. Upon increasing the launched pump power, the erbium-related luminescence in all modes increases linearly (data not shown), demonstrating that the emission observed in Fig. 3(a) is due to spontaneous emission.

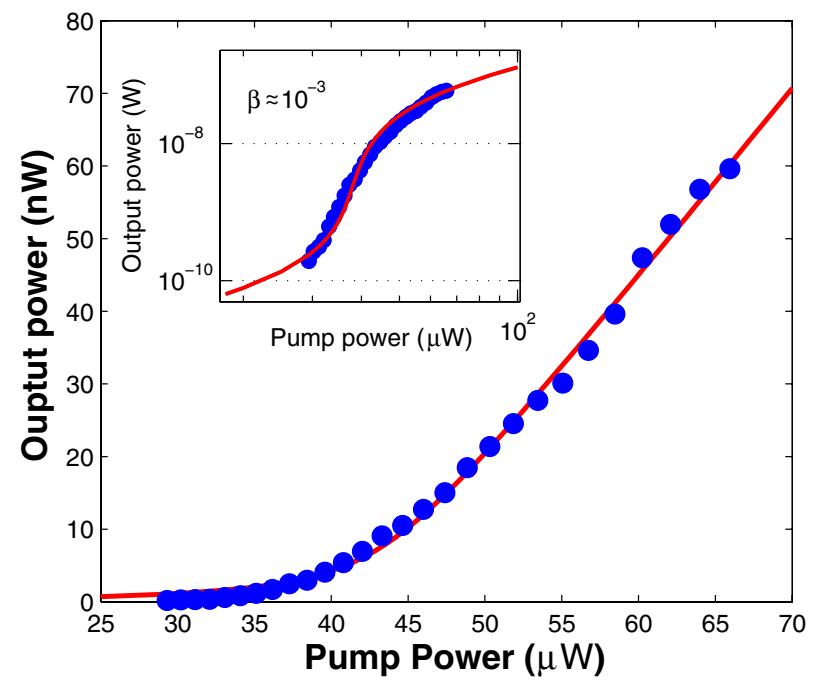

FIG. 4. (Color online) Output power vs launched pump power for the Er-doped microdisk in Fig. 2 (pump wavelength $1480 \mathrm{~nm}$ ). The transition from spontaneous emission to stimulated emission is gradual, indicative that a sizable fraction of the spontaneous emission is coupled into the microcavity. The solid line is a fit using the model from Ref. [17] yielding a lasing threshold of $43 \mu \mathrm{W}$. The inset shows the same data on a double-logarithmic scale with a logarithmic fitting routine which improves fitting for the low-power data and yields a spontaneous emission coupling factor $\beta \approx 1$ $\times 10^{-3}$.

Upon further increase in launched pump power superlinear behavior in the spontaneous emission is observed, as plotted in Fig. 4, followed by a linear pump-output relationship of one of the modes at high power [cf. Fig. 3(b)], which we attribute to lasing. This change is accompanied by an increase in differential slope efficiency for the lasing mode. The remaining (nonlasing) modes did not show this transition. This behavior is well known in microcavity lasers, which due to a large spontaneous emission coupling factor exhibit a gradual transition from spontaneous emission to stimulated emission [17]. From linear interpolation the lasing threshold was estimated to be $43 \mu \mathrm{W}$. This threshold value is consistent with the predicted value [18] using $\mathrm{V}_{m}=2 \pi R A_{m} \approx 200\left(\frac{\lambda}{n}\right)^{3}$, $N_{T}=3.8 \times 10^{20} \mathrm{~cm}^{-3}, \quad \lambda_{p}=1480 \mathrm{~nm}, \quad \lambda_{s}=1555 \mathrm{~nm}, \quad$ and $\Lambda=0.3$ and assuming that the intrinsic $Q$ factor of the lasing mode is $Q_{s}=6 \times 10^{5}$. The emission was observed to be single mode for pump powers up to $400 \mu \mathrm{W}$, and the highest output power observed was $10 \mu \mathrm{W}$. As we have demonstrated for toroidal cavities [18] higher output powers can be achieved for overcoupled conditions, but at the expense of a higher pump threshold.

To determine the spontaneous emission coupling factor $(\beta)$, defined as the fraction of spontaneous emission coupled into the lasing mode with respect to the spontaneous emission into all modes, the input-output power relationship was modeled using a rate equation model [17], which yields

$$
s=\frac{-1}{2 \beta}+\frac{p}{2 \Omega}+\frac{\sqrt{(\beta p-\Omega)^{2}+4 p \beta^{2} \Omega}}{2 \Omega \beta} .
$$

Here $s$ is the cavity photon number, $p$ the pump rate (of $\mathrm{Er}$ ions) and $\Omega$ the cavity loss rate. The solid line in Fig. 4 is a 
three-parameter fit used in Eq. (1), fitting $s$ to the measured output power. The pump coupling efficiency from the fiber into the cavity is assumed unity. The fit exhibits satisfactory agreement with the data. It is noted, however, that this model can deviate from the observed dependence pump-output relations close to the threshold, for several reasons. First, the evanescent waveguide coupling renders the coupled power sensitive to the intrinsic cavity $Q$, which due to the presence of Er is varying. Such a pump-power-dependent $Q$ (due to a pump-induced reduction of Er absorption or nonlinear effects) has already been observed in Raman microcavity lasers $[3,19]$. Specifically the coupled power $(\Delta P)$ is given by $\Delta P=\left[1-\left(\frac{Q_{i}-Q_{e x}}{Q_{i}+Q_{e x}}\right)^{2}\right] P$ where $P$ denotes the launched power and $Q_{i}=\left(1 / Q_{0}+1 / Q_{s}^{\mathrm{Er}}\right)^{-1}$ is the total quality factor at the pump wavelength, which contains a contribution from erbium absorption $\left(Q_{s}^{\mathrm{Er}}\right)$ and other cavity loss mechanisms $\left(Q_{0}\right)$. The former is given by $1 / Q_{p}^{\mathrm{Er}}=\frac{\lambda}{2 \pi n}\left[-\frac{N_{2}}{N_{T}}\left(\alpha_{p}+g_{p}\right)+\alpha_{p}\right]$ where $\left(\alpha_{p} \equiv \Lambda N_{T} \sigma_{p}^{e}, g_{p} \equiv \Lambda N_{T} \sigma_{p}^{a}\right)$ are the Gilles factors describing gain and loss at the pump wavelength [17] and the normalized upper-state population is given (below threshold) by $\frac{N_{2}}{N_{T}}=\frac{\alpha_{p} \phi_{p}}{\phi_{p}\left(\alpha_{p}+g_{p}\right)+N_{T}\left(1 / \tau_{\mathrm{EF}}\right)}$ (where $\phi_{p}$ is the pump photon flux and $\tau_{\mathrm{Er}}$ the erbium upper state lifetime). For low pump powers $1 / Q_{p}^{\mathrm{Er}}=\frac{\lambda}{2 \pi n} \alpha_{p}$, whereas for high pump powers the $Q$ increases to $1 / Q_{p}^{\mathrm{Er}}=\frac{\lambda}{2 \pi n}\left[-\left(\frac{N_{2}}{N_{T}}\right)_{\text {thresh }}\left(\alpha_{p}+g_{p}\right)+\alpha_{p}\right]$ (which can be close to transparency [17]). Since the Er absorption is observed to influence the total cavity $Q$ (cf. Fig. 2), it is clear the pumping-dependent $Q$ will lead to loading effects. As the observed $Q$ factors in the Er absorption band are limited by Er absorption for low pump powers, this effect will thus lead to an increased intrinsic $Q$. Effects of varying $Q$ are most prominent around the threshold, since clamping of the population occurs above threshold, leading to constant $Q$ as is the case for low pump powers. Thus the low- and high-power laser dynamics are well captured by the above model. A second effect that is not taken into account by the above model is a pump power-dependent Er excited-state lifetime, due to cooperative upconversion between excited Er ions [6]. To obtain an improved fit in the region of low pump and high pump power which determines the value of $\beta$ (and in which the aforementioned effects are negligible), the pump-output relationship was fitted on a double-logarithmic scale as shown in the inset of Fig. 4. From the model we derive the spontaneous emission coupling factor, which describes the fraction of spontaneous emission coupled into cavity modes: $\beta=1 \times 10^{-3}$.

We ascribe this relatively low value of $\beta$ to a number of effects. First of all, $\mathrm{Er}^{3+}$ ions in glass exhibit large homogeneous broadening. As a consequence the erbium population can decay via a large number of cavity modes. From the spontaneous emission spectrum in Fig. 2(a), we can estimate that the total number of cavity modes $(N)$ to which the Er ions couple is $\sim 20(\sim 10$ modes at each TE and TM polarization). Consequently, even in the case of spontaneous emission taking place only in cavity modes the $\beta$ which can be expected is $<5 \%(=1 / N)$. The yet lower experimentally observed value is ascribed to the fact that spontaneous emission also occurs into free-space modes (noncavity modes).

In summary, we have realized an erbium-implanted microlaser using a silica microdisk on a silicon wafer. A pump threshold as low as $43 \mu \mathrm{W}$ is observed, and the spontaneous emission coupling factor is determined to be $\sim 1 \times 10^{-3}$ for the lasing mode, in fair agreement with theory. The disk geometry presents several advantages over previous toroidal geometries as it enables direct use of ion implantation, a planar technology, for doping optical microcavities. The CMOS compatibility of all fabrication steps, including ion implantation, may enable the use of these microdisk lasers in photonic and optoelectronic components on a Si chip. Furthermore, these cavities are ideal microlaboratories to study fundamental effects of a broad range of ion-beam-doped optical materials.

This work was supported by DARPA and the Caltech Lee Center for Advanced Networking. T.J.K. acknowledges the Caltech Center of the Physics of Information for support. The Dutch part of this work is part of the research program of FOM which is financially supported by NWO. The authors thank Ali Dabirian from the MPQ for the finite-element numerical modeling.
[1] K. S. Min et al., Appl. Phys. Lett. 69, 2033 (1996).

[2] M. Zacharias et al., Appl. Phys. Lett. 80, 661 (2002).

[3] T. J. Kippenberg, S. M. Spillane, D. K. Armani, and K. J. Vahala, Opt. Lett. 29, 1224 (2004).

[4] H. S. Rong et al., Nature (London) 433, 292 (2005).

[5] A. Polman et al., Appl. Phys. Lett. 84, 1037 (2004).

[6] A. Polman, J. Appl. Phys. 82, 1 (1997).

[7] D. K. Armani et al., Nature (London) 421, 925 (2003).

[8] V. B. Braginskii, V. S. Ilchenko, and M. L. Gorodetskii, Usp. Fiz. Nauk 160, 157 (1990).

[9] V. Sandoghdar, F. Treussart, J. Hare, V. LefevreSeguin, J. M. Raimond, and S. Haroche, Phys. Rev. A 54, R1777 (1996).

[10] L. Yang, D. K. Armani, and K. J. Vahala, Appl. Phys. Lett. 83, 825 (2003).

[11] P. G. Kik, M. L. Brongersma, and A. Polman, Appl. Phys. Lett. 76, 2325 (2000).
[12] T. J. Kippenberg, S. M. Spillane, D. K. Armani, and K. J. Vahala, Appl. Phys. Lett. 83, 797 (2003).

[13] S. L. McCall, A. F. J. Levi, R. E. Slusher, S. J. Pearton, and R. A. Logan, Appl. Phys. Lett. 60, 289 (1992).

[14] S. M. Spillane, T. J. Kippenberg, O. J. Painter, and K. J. Vahala, Phys. Rev. Lett. 91, 043902 (2003).

[15] T. J. Kippenberg, S. M. Spillane, and K. J. Vahala, Opt. Lett. 27, 1669 (2002).

[16] D. S. Weiss, V. Sandoghdar, J. Hare, V. Lefevreseguin, J. M. Raimond, and S. Haroche, Opt. Lett. 20, 1835 (1995).

[17] H. Yokoyama and S. D. Brorson, J. Appl. Phys. 66, 4801 (1989).

[18] B. K. Min, T. J. Kippenberg, L. Yang, K. J. Vahala, J. Kalkman, and A. Polman, Phys. Rev. A 70, 033803 (2004).

[19] B. K. Min, T. J. Kippenberg, and K. J. Vahala, Opt. Lett. 28, 1507 (2003). 